\title{
THE OPTICAL SPECTRA OF V803 CEN*
}

\author{
S.O.Kepler \\ Instituto de Fisica \\ Universidade Federal do Rio Grande do Sul, \\ J.E.Steiner and F.Jablonski \\ Instituto Nacional de Pesquisas Espaciais, Brazil
}

ABSTRACT.

We obtained spectrophotometry of V803 Cen in both high state $(B \approx 13.5)$ and low state $(B \approx 17)$ from $3800 \AA$ to $7100 \AA$ and $5 \AA$ resolution. Essentially all the observed absorption lines are due to He I. No emission lines in either high or low state were detected, as well as no significant departures from a Planck law distribution.

\section{INTRODUCTION}

V803 Cen, also named AE-1, was discovered by Elvius (1975) as a blue variable star with a 4 magnitude luminosity variation on a timescale of days. E.L.Robinson, on a literature search for stars similar to AM CVn (HZ29) and PG1346+082, both interacting binary white dwarfs (IBWD), suggested (private communication) $\mathrm{AE}-1$ as a possible similar star, prompting a search for short timescale variations similar to the ones observed in AM CVn and PG1346+082 (Wood et. al. 1987). These short timescale variations were found by Kepler (1987) and O'Donoghue, Menzies and Hill (1987), demonstrating that V803 Cen was indeed similar to the IBWD PG1346+082.

Since all the spectral observations were obtained with V803 Cen in either high state or medium state, and also only observed in the blue (Elvius 1975, Westin 1980, and O'Donoghue, Menzies and Hill 1987), we decided to obtain spectrophotometry of the star from 3800 to $7100 \AA$ to see if we could detect light from different components of the system, as well as to see if we could detect any emission line, especially on the low state.

Our observations show no evidence of emission lines or light from more than one component in the spectra at low or high state.

\section{OBSERVATIONS}

We obtained three spectra of V803 Cen at CTIO, using the '2D-Frutti' two- dimensional photoncounting detector on the Boller and Chivens spectrograph of the $1.0 \mathrm{~m}$ telescope, with a 300 lines $\mathrm{mm}^{-1}$ grating. The wavelenght coverage was from 3800 to $7100 \AA$, and the resolution was $\approx 5 \AA$ (FWHM). A WG360 filter was used to minimize contamination by second-order blue light in the red, and the observations were done with a 5 arcsec slit. We observed at least one spectrophotometric standard every night.

Data reduction was done with IRAF, following a standard procedure; the two- dimensional images were first corrected for detector distortions using a calibration spectra of a tungsten lamp taken through a multihole decker and a long-slit HeAr lamp. After subtracting the dark current scaled by the exposure time, the images were divided by a normalized flatfield image obtained from dome-flat exposures. Sky-subtracted spectra were then obtained, rebinned onto a final linear wavelenght scale using the calibration derived from a HeAr lamp exposure taken at the position of the star, and corrected for extinction using the mean curve given by Stone and Baldwin (1983).

The three exposure obtained were:

1) $\mathrm{d} 001,1600 \mathrm{sec}$, on 26.06 .86 , UT $23: 08$

2) d002, $600 \mathrm{sec}$, on 02.07 .86 , UT $23: 15$

3) d003, $10800 \mathrm{sec}$, on 20.07 .87 , UT $0: 59$

$10800 \mathrm{sec}$, on 21.07 .87 , UT $0: 55$. low state.

Exposure d003 is the sum of two exposures obtained on consecutive days in which the star stayed in

* Based on observations obtained at Cerro Tololo Inter-American Observatory, National Optical Astronomy Observatories, operated by the Association of Universities for Research in Astronomy, Inc., under contract with the National Science Foundation. 
Figure 1 Spectrophotometry of V803 Cen from high state $(B \approx 13.5)$, to low state $(B \approx 17.2)$.

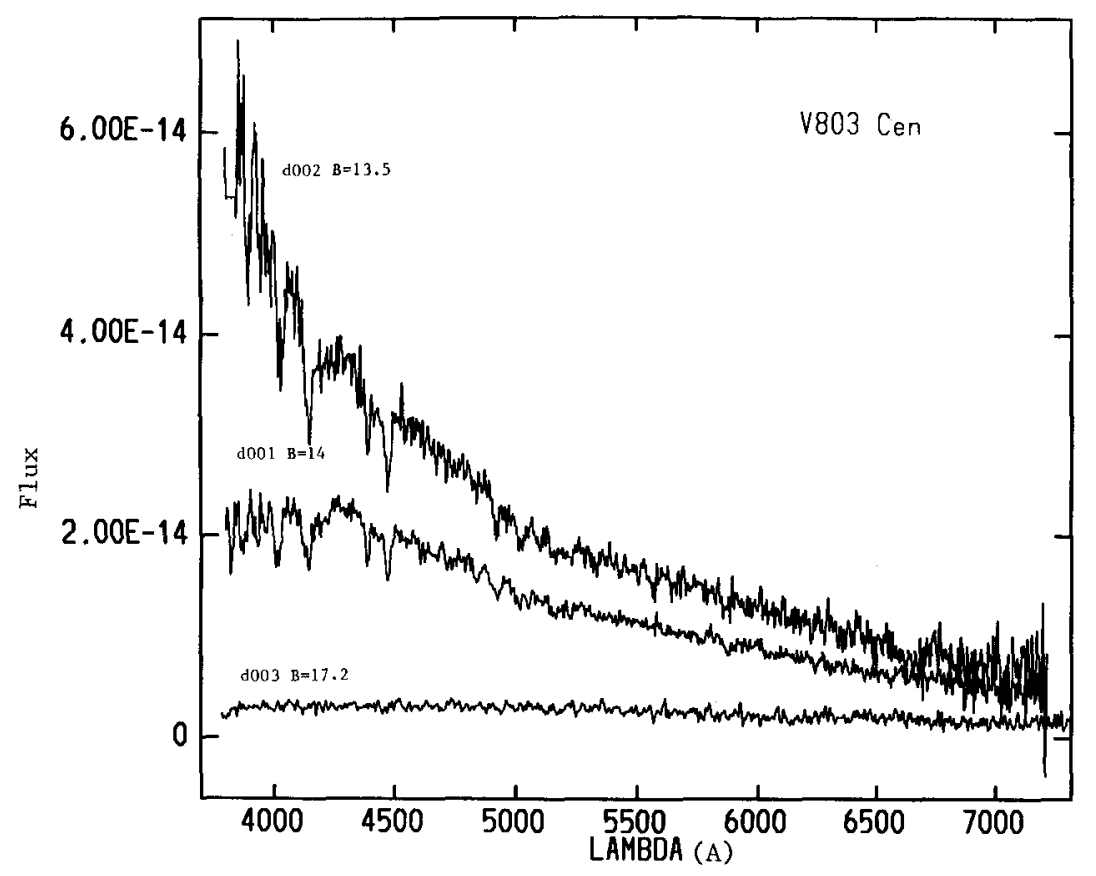

Figure 2 Line identification for the spectra of V803 Cen at high state (d001 - $B \approx 14$ ). All the lines identified bellow the spectra are He I lines. The lines within parenthesis are unidentified. The data has been smoothed with a 3 point boxcar average for presentation.

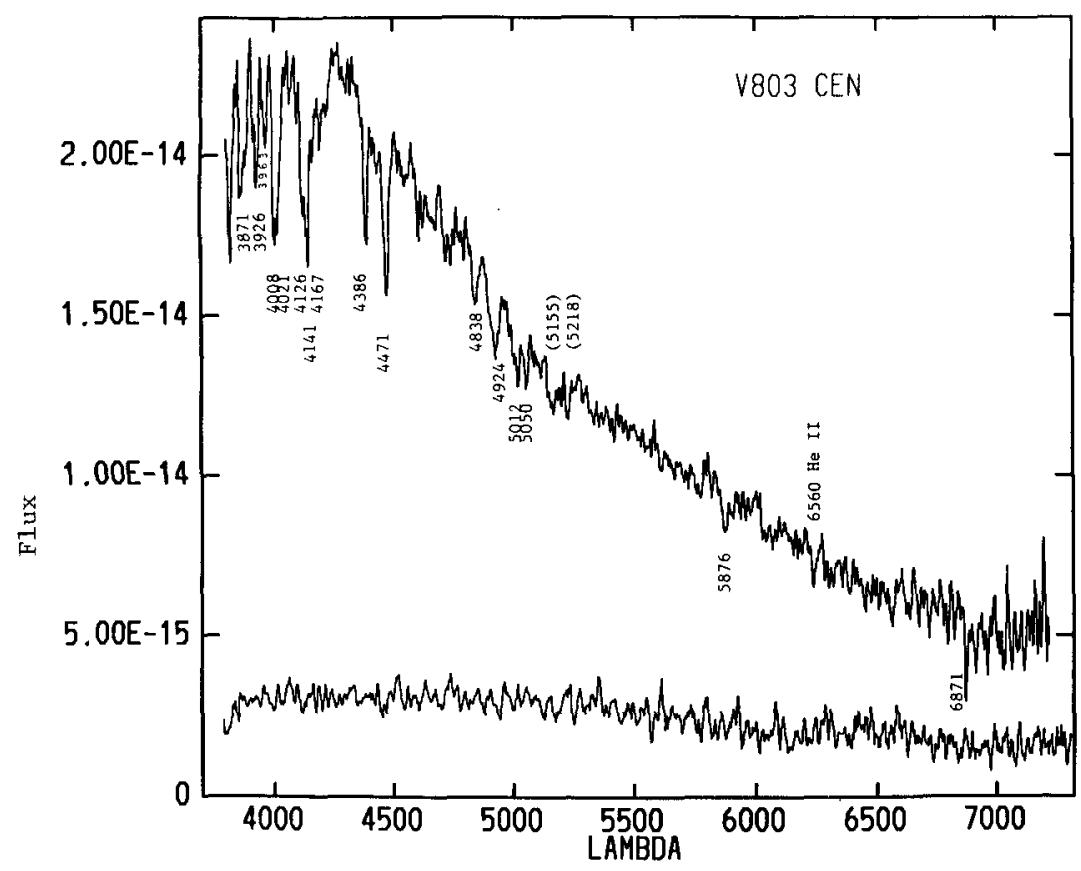




\section{DATA ANALYSIS}

The high state spectra contains only He I lines, with a possibility for a He II line at $6560 \AA$. A fit of a Planck function to the portion of the spectra redward of $4500 \AA$ for $\mathrm{d} 001$, when the count rate corresponded to $B \approx 14$, gives an effective temperature of $T_{\text {eff }}=17106 \pm 275 \mathrm{~K}$. The curvature of the spectra blueward of $4500 \AA$ could be caused by undercorrection with the spectrophotometric standard, since we only observed one standard that night, or possibly by a strong blanketing effect due the He I lines. There is no evidence for either a blue or red excess continuum emission.

For d002, at a high state around $B \approx 13.5$, a fit of a Planck function from $3900 \AA$ to $7100 \AA$ gives $T_{\text {eff }}=25776 \pm 682 \mathrm{~K}$, and also shows no evidence of excess continuum. The temperatures at high state $\left(T_{\text {eff }} \leq 30000 \mathrm{~K}\right)$ are still consistent with the absence of strong He II lines, especially $\lambda 4686$.

The low state spectra $\mathrm{d} 003$, at $B \approx 17.2$, unfortunately of low $\mathrm{S} / \mathrm{N}$ due to the small size of the telescope (obtained with a small telescope because we cannot predict what will be the magnitude of the star at any given period), shows no evidence for lines, either in absorption or emission. A Planck function fit to the whole spectrum gives an effective temperature of $T_{e f f}=7315 \pm 73 K$. The low state spectra also does not show any evidence for multiple components.

In conclusion, the observed spectra of V803 Cen shows essentially only He I lines, shallow in comparison with that of a DB white dwarf, and does not show any evidence for multiple components. The observations are therefore consistent with the model for the interacting double degenerate star, with two Helium white dwarfs and an optically thick accretion disk. The 4 magnitude luminosity variations detected in the star can, in the IBDW model, be explained by mass transfer between the two white dwarfs. The absence of multiple components in the observed spectra indicates that all the optical light comes from the accretion disk. The absence of emission lines even at low state implies that, unless we are seeing a $7300 \mathrm{~K}$ white dwarf, the disk must be optically thick even in faint state.

This work was partially supported by grants from CNPq and FINEP - Brazil.

\section{REFERENCES}

Elvius, A. 1975, Astr. Astrophys., 44, 117.

Kepler, S.O. 1987, IAU Circular No. 4392.

O'Donoghue, D., Menzies, J.W., and Hill, P.W. 1987, Mon. Noi. R. Astr. Soc., 227, 347.

Stone, R.P.S., and Baldwin, J.A. 1983, Mon. Not. R. Astron. Soc., 204, 347.

Westin, B.A.M. 1980, Astr. Astrophys., 81, 74.

Wood, M.A., Winget, D.E., Nather, R.E., Hessman, F.V., Liebert, J., Kurtz, D.W., Wesemael, F., and Wegner, G. 1987, Astrophys. J., 313, 757.

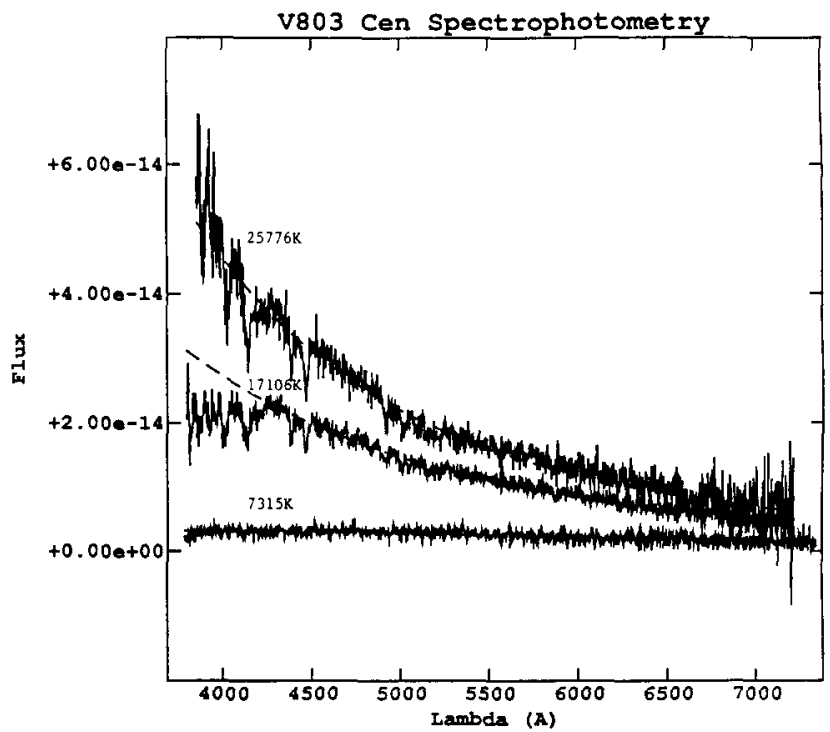

Figure 3 Planck function fits to the spectra of V803 Cen. The dashed line is a plot of only one Planck function to each spectrum. The curvature blueward of $4500 \AA$ in d001 is probably instrumental. 